\title{
Tendencias y buenas prácticas en la atención a la infancia en situación de riesgo social
}

\section{SIIS Centro de Documentación y Estudios}

<estudios@siis.net>

\begin{abstract}
Ebidentzia zientifikoak agerian jarri du haurtzaroan sorturiko desberdintasunek ondorioak izaten dituztela pertsonen bizitza osoan, eta hertsiki loturik daudela pobrezia eta desberdintasunaren belaunaldien kopiaketan. Halaber, ingurune sozial eskasetatik datozen adingabekoen egoera desabantailatsuak orekatzera bideratutako politikek agertu dute itzulkin ekonomiko eta sozial positiboak dituztela, eta modu horretan ekiditen direla biztanleriaren talde horren baitan eragin jasoa duten arazo sozial horiek gertatzea edota are gehiago okertzea. Artikulu honek eskaintzen du panorama orokor bat 'haurtzaroaren errefortzurako' politiketako jardueren arlo nagusi eta helburuekiko, eta horretarako biltzen dira maila nazionalean eta nazioartean ezarri diren jarduera egokiak.
\end{abstract}

\section{GAKO-HITZAK:}

Haurtzaroa, pobrezia, hezkuntza bitarteko gizarteratzea, prebentzioa, arreta goiztiarra, familiaeskuartzea.
La evidencia científica ha puesto de manifiesto que las desigualdades generadas en la infancia tienen consecuencias a lo largo de todo el ciclo vital de las personas, y están estrechamente vinculadas al fenómeno de la reproducción intergeneracional de la pobreza y la desigualdad. Además, las políticas dirigidas a compensar las situaciones de desventaja de los y las menores procedentes de entornos socialmente desfavorecidos han demostrado tener un retorno económico y social positivo, logrando evitar la aparición o el agravamiento de problemas sociales con una alta incidencia en estos grupos de población. Este artículo ofrece un panorama general de las principales áreas de actuación y de los objetivos de las políticas de 'refuerzo en la infancia', a través de una recopilación buenas prácticas implementadas a escala nacional e internacional en este ámbito.

\section{Palabras Clave:}

Infancia, pobreza, inclusión educativa, prevención, atención temprana, intervención familiar. 


\section{Introducción}

Proceder de un contexto familiar vulnerable afecta de forma decisiva a las oportunidades vitales de niños y niñas. Un punto de partida desfavorable en la infancia se relaciona con un menor acceso a la formación y la cultura, un bajo rendimiento escolar, peores índices de salud física y mental, y una mayor probabilidad de adoptar conductas de riesgo y comportamientos antisociales a lo largo de la vida (Assiego y Ubrich, 2015; Harvey, 2014; Allen, 2011; Waldfogel, y Washbrook, 2011; Field, 2010). Las desigualdades generadas en la infancia, además, tienen consecuencias a lo largo de todo el ciclo vital de las personas, dando lugar a una reproducción o transmisión intergeneracional de la pobreza y la exclusión social (Fundación Foessa, 2016; Unicef, 2014; Field, 2010).

En este sentido, las políticas de refuerzo en la infancia no sólo contribuyen a promover la igualdad de oportunidades en edades tempranas, sino que existe una evidencia científica creciente de que tienen un retorno económico y social positivo. Numerosos estudios han demostrado que el abordaje tardío de los problemas de aprendizaje, integración y conducta de niños y niñas resulta, no sólo más costoso, sino más ineficaz, un hecho que sitúa a las políticas de refuerzo en la infancia entre las políticas de inversión social (Allen, 2011; Field, 2010; Karoly, Kilburn y Cannon, 2005).

El principal objetivo de las políticas de refuerzo en la infancia es contribuir al desarrollo integral de los niños y niñas en situación de desventaja social. Desde el punto de vista de su contenido, las intervenciones se centran en los siguientes ámbitos:

- La provisión de servicios de atención temprana y detección precoz dirigidos a ofrecer apoyo suplementario a las familias desde el momento del nacimiento y durante los primeros años de vida del menor (de o a 6 años), una fase crítica del desarrollo que tiene un efecto de largo alcance en las capacidades cognitivas, emocionales y sociales de los niños y niñas en etapas posteriores de la infancia, la adolescencia y la edad adulta.

- La mejora de las habilidades parentales de los padres y madres para hacer frente a las distintas etapas de la crianza de sus hijos/as, especialmente, mediante la implementación de programas de parentalidad positiva y servicios de orientación o asesoría en situaciones de conflicto familiar.

- La promoción de la salud física y mental de los y las menores, a través de:

- Servicios y programas de salud maternoinfantil dirigidos a garantizar unas buenas condiciones de salud y bienestar de los menores y sus madres durante los primeros años de vida.
- Programas que garanticen el acceso a servicios (logopedia, psicomotricidad) y bienes (gafas, audífonos) relacionados con la salud de los menores que no están cubiertos por el sistema de salud pública.

- Programas que impulsen hábitos de vida saludables en la familia en ámbitos como la alimentación, la higiene o la actividad física.

- Programas dirigidos a promover el desarrollo de competencias sociales y emocionales de niños y niñas.

- La mejora del rendimiento escolar de los niños y niñas mediante:

- Programas dirigidos a promover la participación y las competencias de padres y madres en el apoyo y acompañamiento a la educación de sus hijos e hijas.

- Programas de refuerzo escolar dirigidos a ofrecer apoyo a los niños y niñas que muestran dificultades de aprendizaje, más allá del horario escolar.

- Programas dirigidos a garantizar el acceso de los niños y niñas a material escolar y otros equipamientos necesarios para participar adecuadamente en el entorno de la educación formal, pero también para crear entornos de aprendizaje en sus respectivos hogares que contribuyan a su estimulación y desarrollo cognitivo.

- Favorecer el acceso de los niños y niñas a programas y actividades culturales, de ocio y tiempo libre:

- Mediante fórmulas subvencionadas de acceso a las actividades culturales, de ocio y tiempo libre para los y las menores, en el marco de la oferta dirigida al conjunto de la población.

- Favoreciendo el acceso de las familias en situación de desventaja social a programas de actividades culturales, ocio y tiempo libre en familia.

- Mediante programas que ofrezcan actividades culturales, de ocio y tiempo libre para los y las menores procedentes de familias en situación de desventaja social los fines de semana y durante los periodos vacacionales.

- Tratar de que los programas incluyan también acciones que contribuyan a mejorar la situación de los padres y madres en aquellas áreas donde presenten una mayor vulnerabilidad (ámbito económico, problemas de salud mental, soledad, aislamiento y falta de redes de apoyo, baja formación, desconocimiento del idioma local, escasas habilidades personales o sociales).

En este artículo, se presentan una serie de programas que han demostrado su eficacia en cada uno de estos ámbitos y se enumeran también algunos factores de éxito identificados en la evaluación de programas similares. 


\section{Detección precoz y atención temprana}

La detección precoz y la atención temprana tienen como objetivo identificar y abordar los problemas relacionados con el aprendizaje, la conducta, la salud $y$, de forma general, el bienestar de niños y niñas de forma temprana, concretamente, en la primera infancia (de o a 6 años). Existe una fuerte evidencia científica de la importancia que el periodo de o a 3 años tiene de cara a sentar las bases del desarrollo y fijar las estructuras cognitivas, la competencia emocional y las habilidades sociales básicas de niños y niñas (Harvey, 2014; Waldfogel y Washbrook, 2010). Esto supone que el entorno en el que los menores viven durante sus primeros años de vida compromete de forma crucial sus posibilidades de desarrollo y puede dar lugar a desigualdades en edades tan tempranas como los 2 y 3 años (Harvey, 2014; Field, 2010; Waldfogel y Washbrook, 2010; Karoly, Kilburn y Cannon, 2005). Como señala Field (2010), el nivel de desarrollo de un menor a los 22 meses sirve como un indicador bastante fiable del nivel educativo que esta persona haya alcanzado a la edad de 26 años.

Las intervenciones en el ámbito de la atención precoz tienen como objetivo incidir sobre los factores que afectan al desarrollo de niños y niñas durante sus primeros años de vida ${ }^{1}$. En la revisión de programas aplicados en el ámbito de la atención precoz realizada por Axford et al. (2015), estos autores clasifican los programas en tres tipos, en función del principal objetivo que guía la intervención:

- Promoción del vínculo y de un apego seguro entre los bebés y sus progenitores.

- Mejora de las habilidades sociales, emocionales y comportamentales de niños y niñas.

- Mejora de las habilidades comunicativas y lingüísticas.

\subsection{Promoción del vínculo y de un apego seguro entre los bebés y sus progenitores}

Este tipo de programas abarcan el periodo perinatal y pueden extenderse desde el embarazo hasta el primer o segundo año de vida del bebé. La mayoría están basados en la teoría del apego y el principal objetivo de las intervenciones es promover una parentalidad sensible a las necesidades y los estados

${ }^{1}$ El desarrollo de niños y niñas durante los primeros años de vida está determinado, principalmente, por los siguientes factores (Harvey, 2014; Field, 2010; Frazer, 2010):

- Una buena nutrición, un buen estado de salud y bajos niveles de estrés de la madre durante el embarazo.

- Una buena nutrición y un buen estado de salud adecuados de la niña o niño durante los primeros años de vida.

- El desarrollo de un vínculo y una relación de apego segura y estable entre la madre y el niño. Este factor se halla estrechamente vinculado al estado de salud de la madre y, especialmente, a su salud mental.

- Un entorno estimulante determinado por la calidad y la frecuencia de la interacción con sus progenitores y otras figuras cuidadoras relevantes, y también por la interacción positiva con otros niños y niñas. internos del bebé, fortaleciendo la capacidad de los progenitores de responder a sus demandas. Algunas de las intervenciones abordan, además, problemas de los padres y madres (depresión, ansiedad, trastornos mentales, adicciones) que interfieren en su capacidad de ofrecer un buen cuidado a sus hijos/as.

\section{Axford et al. (2015) identifican diversas}

formas de provisión de estos programas para grupos de población en situación de riesgo: visitas domiciliarias, sesiones individuales de retroalimentación a partir de grabaciones de vídeo en las que se analiza la interacción progenitor-bebé, y sesiones grupales de formación e información. La revisión realizada por estos autores muestra que los programas de visitas domiciliarias - donde un profesional cualificado acude regularmente al domicilio familiar para trabajar con los progenitores aspectos relacionados con el cuidado y la crianza del bebé- son los más eficaces para los grupos de alto riesgo (madres adolescentes, familias monoparentales en situación de pobreza, mujeres embarazadas con trastorno depresivo o síntomas de tenerlo) ${ }^{2}$. Uno de los programas de este tipo que ha demostrado mayor eficacia es el Nurse Family Partnership (NFS) ${ }^{3}$, aplicado inicialmente en los Estados Unidos y dirigido a madres adolescentes menores de 19 años. En el caso de los grupos de población vulnerable que no muestran claros indicadores de riesgo, los programas grupales (Baby Steps, Mellow Bumps) o los basados en sesiones individuales (Video Feedback Intervention to Promote Positive Parenting [VIPP]) han demostrado ser intervenciones adecuadas y suficientes. Finalmente, también existen programas de psicoterapia específicamente dirigidos a niños y niñas con problemas de conducta a edades tempranas ${ }^{4}$.

${ }^{2}$ Existen programas de visitas domiciliarias de carácter preventivo o de screening, cuyo objetivo es identificar casos en los que, en ausencia de indicadores de riesgo en la unidad familiar, los progenitores tengan, no obstante, dificultades para llevar a cabo la crianza de sus hijos/as. Recientemente, en el marco del Healthy Child Programme, en el Reino Unido han comenzado a realizarse visitas domiciliarias, de carácter obligatorio, a todos los hogares donde reside algún menor de entre o y 2 años de edad.

${ }^{3}$ En la revisión de Axford et al. (2015), se identifican ocho programas de atención temprana implementados a través de visitas domiciliarias y cuyos resultados han demostrado ser positivos en evaluaciones hechas con grupos de control, aunque la solidez de las evaluaciones y la magnitud del efecto observado varía ampliamente de un programa a otro y entre los diversos estudios realizados. Estos programas son los siguientes: Community Mothers, Early Start, Family Thriving Programme, Nurse Family Partnership, Maternal Early Childhood Sustained Home Visiting (MECSH), Nobody Slips through the Net, SafeCare y The Social Baby.

4 Estos programas se aplican tras la detección de problemas de conducta en los menores a edades tempranas. En la revisión de Axford et al. (2015), se recogen cinco programas de este tipo. Cuatro de ellos obtienen resultados positivos en evaluaciones realizadas con grupos de control (Anna Freud Parent Infant Project [PIP]; Toddler-Parent Psychotherapy; Watch, Wait, Wonder; y Child Parent Psychotherapy [modelo Lieberman]) y las evaluaciones identifican una mejora en los síntomas de los menores, en su comportamiento, en los niveles de estrés de las madres, así como una reducción de las formas de apego inseguro y apego desorganizado. 
Cuadro 1. Family-Nurse Partnership (Estados Unidos)

El programa Family-Nurse Partnership tiene como objetivo mejorar el bienestar de las madres jóvenes primerizas con bajos ingresos y sus hijos/as.

\section{Objetivos de la intervención}

- Mejorar los hábitos de salud de la madre (consumo de tabaco, alcohol y otras drogas), el autocuidado y la realización de los controles prenatales necesarios durante el embarazo.

- Optimizar el estado de salud y el desarrollo de los y las menores durante los dos primeros años de vida, capacitando a los padres en la provisión de un cuidado más responsable y competente.

- Mejorar la situación económica de las familias a través de la planificación familiar, el acceso de los progenitores a formación y la promoción del acceso a un empleo estable.

\section{Grupo al que se dirige la intervención}

Madres jóvenes (especialmente, menores de 19 años) primerizas que se encuentran en el primer o segundo trimestre del embarazo y se hallan en situación de pobreza o bajos ingresos.

\section{Planteamiento que subyace a la intervención}

El programa se basa en una serie de asunciones que determinan, por un lado, el grupo de población al que se dirige y, por otro, la forma de provisión:

- El primer embarazo es el mejor momento para inculcar buenos hábitos de salud y buenas competencias parentales en los progenitores.

- El establecimiento de una relación de confianza entre la madre y el/la enfermero/a que la atiende en el marco del programa es un factor crítico para asegurar el éxito de la intervención.

- Esta relación debe centrarse en la promoción de la autoeficacia de la madre para el cuidado de sus hijos/as y debe durar el tiempo suficiente como para capacitar a los padres y madres en el ejercicio de una parentalidad positiva durante algunos de los periodos más críticos del desarrollo de niñas y niños de edad temprana (de o a 2 años).

- El programa se centra en objetivos pequeños que los progenitores pueden alcanzar entre una y otra visita domiciliaria.

\section{Forma de provisión del programa}

El programa se articula mediante visitas domiciliarias realizadas por un/a enfermero/a. Estas visitas se extienden desde el periodo del embarazo hasta que el/la niño/a cumple los dos años de edad. El profesional actúa como un profesional de referencia, de modo que, idealmente, debe mantenerse durante todo el tiempo que dura la intervención. Los/as enfermeros/as que participan en el programa reciben una formación específica de dos semanas antes de incorporarse. La frecuencia de las visitas es, en un principio, quincenal, y se va espaciando a medida que avanza el programa. No obstante, la frecuencia debe ajustarse a las necesidades de cada familia, pudiendo llegar a realizarse visitas semanales en los casos en que sea necesario.

El objetivo de las visitas domiciliarias es el siguiente:

- Durante el embarazo, mejorar los hábitos de salud de la madre y capacitarla para detectar los problemas y complicaciones que puedan surgir. Además, también se ayuda a la madre a ampliar su red de apoyo social y familiar, en caso de que sea escasa, con el objetivo de que pueda recurrir a ella tras el nacimiento del niño o la niña.

- Tras el nacimiento del hijo/a, las visitas se centran en trabajar aspectos relacionados con el cuidado, el buen trato, y la estimulación cognitiva y afectiva del bebé.

- Finalmente, un objetivo transversal de la intervención es detectar los recursos sociales y comunitarios (formación, empleo, servicios de salud, servicios de cuidado) de los que podrían beneficiarse los progenitores, y actuar como puente para que las personas accedan a ellos.

\section{Coste del programa}

El coste medio anual de la implementación es de 3.200 \$ por familia durante la primera fase del programa, debido a los costes vinculados a la formación de los profesionales implicados, así como a la menor eficiencia de las intervenciones durante la fase de aprendizaje y el periodo de ajuste del programa. Este coste debe calcularse para los primeros tres años en los que el programa está en marcha. Posteriormente, su coste desciende hasta los 2.800 \$ por familia.

\section{Resultados}

El programa ha sido objeto de numerosos estudios que han analizado su eficacia, ya que fue implementado por primera vez en 1977. El estudio más reciente recogido en el portal Child Trends (〈https://www.childtrends.org〉). arroja los siguientes resultados referentes a niñas y niños que han sido objeto de intervención en el programa:

- Los menores participantes demostraban puntuaciones más elevadas en funcionamiento intelectual $(92,34)$, lenguaje receptivo $(84,32)$ y capacidad aritmética $(88,61)$, en comparación con el grupo de control $(90,24 ; 82,13$ y 85,42, respectivamente) a la edad de 6 años.

- El porcentaje de madres que señalaron que sus hijos/as mostraban algún tipo de problema grave de conducta a la edad de 6 años fue de 1,6\% en el grupo de intervención y de 5,4\% en el grupo de control.

- No se observa, sin embargo, ningún efecto en el comportamiento internalizado y externalizado de niñas y niños, en la capacidad empática, ni tampoco en el comportamiento reportado por el profesorado en el ámbito escolar a la edad de 6 años. 
2.2. Mejora de las habilidades sociales, emocionales y comportamentales de niños y niñas

Este tipo de programas se dirigen, por lo general, a niños y niñas de más de 2 años, y se centran en conseguir una mejora del comportamiento y el funcionamiento social y emocional de los menores. Son una modalidad específica de los programas de parentalidad positiva, pero enfocados a la intervención con niños y niñas de entre los o y los 6 años. El apartado 3 de este artículo analiza específicamente este tipo de programas.

\subsection{Mejora de las habilidades comunicativas y lingüísticas}

Un tercer tipo de intervención en el ámbito de la atención temprana se dirige a potenciar o compensar el retraso en el desarrollo de habilidades comunicativas y lingüísticas de los menores. Garantizar el acceso a servicios de educación infantil de calidad para los menores en situación de vulnerabilidad social constituye una de las principales medidas que se recomiendan a este respecto (Frazer, 2016; Assiego y Ubrich, 2015; Field, 2010). No obstante, la evidencia científica muestra el papel crucial que las familias desempeñan en la promoción de estas habilidades (Waldfogel y Washbrook, 2010; Stormshak, Connell y Dishion, 2009). Los principales factores de éxito asociados con las intervenciones dirigidas a mejorar el desarrollo cognitivo y el rendimiento escolar de los y las menores en situación de desventaja social se analizarán en el apartado 5. No obstante, se mencionan aquí algunos programas específicamente enfocados a la primera infancia que han demostrado ser eficaces.

La revisión hecha por Axford et al. (2015) recoge programas de prevención universal, así como programas específicamente dirigidos a familias en situación de riesgo. En este último caso, las evaluaciones muestran que los mejores resultados se obtienen en programas que se basan exclusivamente en visitas domiciliarias, o bien en aquellos que combinan las sesiones grupales dirigidas a padres, madres u otras figuras educativas relevantes (abuelos/as, tíos/as, cuidadores/as) con actividades de aprendizaje dirigidas a niñas y niños, y algunas visitas domiciliarias en las que se trabaja individualmente con las familias. Además, en ocasiones, las sesiones grupales se estructuran en dos partes. En la primera, los progenitores y los/as niños/as desarrollan actividades de forma separada $y$, después, se fomenta la interacción entre ambos y la puesta en práctica de los contenidos trabajados en la sesión.

Los programas que se estructuran en torno a visitas domiciliarias tienen una duración de entre tres meses y dos años, en función del programa. Existen también importantes diferencias en la duración de las visitas (entre treinta minutos y dos horas). Las evaluaciones muestran resultados positivos para la mayoría de los programas analizados, y el efecto más significativo se asocia con dos de estos programas: Playing and Learning Strategy (PALS) ${ }^{5}$ y Let's Play in Tandem ${ }^{6}$.

En cuanto a las intervenciones basadas en la combinación de visitas domiciliarias y sesiones grupales, la revisión de Axford et al. (2015) halla resultados positivos para seis de ellas a partir de evaluaciones con grupos de control?. Concretamente, las evaluaciones identifican una mejora en la implicación de los padres y madres en la educación y el proceso de aprendizaje de sus hijos/as, una mejora de las estrategias parentales para acompañar la educación y desarrollo de sus hijos/as, y la reducción de los comportamientos punitivos hacia los menores. Entre los resultados asociados al desarrollo de niñas y niños, se observa una mejora de las habilidades lingüísticas, la capacidad de lectura y la capacidad numérica.

\section{La adquisición de competencias parentales y los programas de parentalidad positiva}

Los programas de parentalidad positiva parten de la idea de que un adecuado ejercicio del rol parental no es algo innato, sino que conlleva el despliegue de habilidades y conocimientos de carácter complejo y debe ser, por lo tanto, objeto de aprendizaje y

5 Playing and Learning Strategy (PALS) es un programa de intervención individualizada estructurado en torno a 10-12 visitas domiciliarias, de unos 90 minutos cada una, llevadas a cabo por educadores familiares. Cuenta con dos versiones, la primera dirigida a menores de entre 5 y 18 meses (PALS I) y la segunda enfocada a niñas y niños de entre 18 meses y 3 años (PALS II). Mientras que la primera versión del programa se centra, sobre todo, en la promoción del vínculo, el apego y la interacción positiva entre los progenitores y sus hijos/as, la segunda ha mostrado resultados significativos en la estimulación verbal de los padres y madres respecto a sus hijos/as, el incremento de actitudes cooperativas en niñas y niños, y el uso y riqueza de vocabulario de los/as menores. El contenido de las sesiones abarca las siguientes cuestiones: a) atender y responder a las señales comunicativas de los bebés; b) responder adecuadamente a las señales positivas y negativas del comportamiento de niñas y niños; c) apoyar el aprendizaje de niñas y niños manteniendo su atención e interés; d) utilizar juegos y actividades para estimular el aprendizaje; e) estimular el lenguaje y los conocimientos básicos sobre los mecanismos de adquisición del lenguaje; f) promover la cooperación y responder al comportamiento negativo de niñas y niños; g) incorporar las estrategias aprendidas para apoyar al desarrollo de niñas y niños a través de las actividades de la vida diaria (las comidas, vestirse, la higiene y el aseo personal, el tiempo de juego).

${ }^{6}$ Let's Play in Tandem es un programa de preparación a la educación obligatoria dirigido a niñas y niños de 3 años procedentes de entornos sociales desfavorecidos. Su objetivo es potenciar el desarrollo cognitivo de los niños y niñas, así como su capacidad de autoaprendizaje. Niñas y niños participan conjuntamente con sus progenitores en actividades de juego, a través de las cuales se adquieren los conocimientos y el vocabulario que se espera que los menores tengan en el momento de acceder al sistema escolar. La duración del programa es de 12 meses y se lleva a cabo mediante visitas domiciliarias semanales en las que un educador familiar dinamiza las sesiones, de entre 90 y 120 minutos de duración. Cada sesión incluye tres actividades dirigidas a la adquisición de vocabulario, de conocimientos generales y habilidades de prelectura, y de habilidades numéricas.

7 Los programas identificados para los que se han hallado resultados positivos son los siguientes: Home Instruction Programme for Preschool Youngsters (HIPPY), Early Head Start, Getting Ready, Sure Start y Raising Early Achievement in Literacy (REAL). Una breve descripción de cada uno de ellos puede encontrarse en Axford et al. (2015). 
entrenamiento. La finalidad de estos programas es mejorar la competencia parental ${ }^{8}$, tanto de forma preventiva - esto es, preparando a los padres y madres para responder adecuadamente a las necesidades y capacidades de sus hijos/as en cada etapa del desarrollo- como en respuesta a conflictos específicos relacionados con la crianza.

Los programas de parentalidad positiva varían en su forma de provisión (autoadministrados, basados en sesiones grupales, sesiones individuales con familias, combinación de sesiones grupales e individuales), la edad de los y las menores a cuyos progenitores se dirigen y su duración, entre muchos otros factores. Existen, no obstante, algunos programas cuya eficacia ha sido ampliamente demostrada y que los expertos señalan como intervenciones efectivas que son aplicables en diversos contextos (Axford et al., 2015; Goff et al., 2013; Marklund y Simic, 2012):

- Programa Triple P: programa de parentalidad positiva estructurado en cinco niveles, en función de la intensidad de apoyo que precisen las familias. Abarca todo el continuo de la atención, desde la prevención universal hasta la intervención en situaciones de graves problemas de conducta de los menores o de disfunción familiar. La intervención directa con las familias se articula a partir del segundo nivel (Selected Triple P) e incluye la posibilidad de hacer breves consultas telefónicas o presenciales sobre problemas de conducta de los menores. El programa de mayor intensidad (Enhanced Triple P) se estructura en un mínimo de once sesiones de una hora de duración, que incluyen sesiones individualizadas de formación en habilidades parentales y visitas domiciliarias.

- Strenghtening Families Programme: es un programa específicamente dirigido a familias en situación de riesgo y aplicable a todos los tramos de edad, dado que cuenta con versiones adaptadas a cada uno de ellos (3-5 años, 6-11 años, 12-16 años). Se estructura en 14 sesiones grupales, a las que padres y madres acuden con sus hijos/as, aunque las sesiones incluyen tanto actividades

${ }^{8}$ Los programas de parentalidad positiva tratan de promover la competencia parental y, a través de ella, la resiliencia del conjunto de la unidad familiar. Rodrigo López, Máiquez y Martín Quintana (2010) definen cuatro áreas de competencia parental:

- Educativa: abarca todas las dimensiones de la relación entre los progenitores y sus hijos/as.

- Agencia parental: hace referencia a las actitudes, percepciones y vivencias de los padres y madres respecto a su rol parental (autoeficacia parental, locus de control interno, acuerdo en la pareja, percepción ajustada del rol parental, satisfacción respecto a la tarea de ser padre/madre).

- Autonomía personal y búsqueda de apoyo social: asunción del rol parental y responsabilidad en el ejercicio de éste. Capacidad para identificar y utilizar recursos sociales y comunitarios que contribuyan al bienestar personal, del niño/a y del conjunto de la unidad familiar.

- Desarrollo personal: hace referencia al nivel de desarrollo personal del padre/madre (control de impulsos, asertividad, autoestima, habilidades sociales, regulación emocional, estrategias de afrontamiento ante situaciones de estrés, resolución de conflictos interpersonales). conjuntas como ejercicios dirigidos a los/as niños/as y a sus progenitores de forma separada.

- Incredible Years: es un programa estructurado en un número variable de sesiones grupales, de entre dos horas y dos horas y media de duración, exclusivamente dirigidas a padres y madres, con la excepción del programa para niños/as menores de 1 año, donde los progenitores acuden a las sesiones junto con sus bebés. El programa se dirige a padres y madres con hijos/as de entre o y 12 años, y cuenta con cinco módulos básicos y una versión avanzada para familias donde niñas y niños presentan graves problemas de conducta 0 la unidad familiar se halla en situación de especial vulnerabilidad. Habitualmente, el programa cubre los gastos relacionados con el transporte, el cuidado de los/as hijos/as y las comidas (almuerzo o merienda), lo cual facilita el acceso de las familias en situación de desventaja social.

Aunque, en un principio, los programas de educación parental fueron diseñados para intervenir con familias en situación de riesgo (padres, madres o hijos/as con problemas de adicción, situaciones de fuerte conflicto familiar), en los últimos años se observa una tendencia a extender los servicios de asesoría u orientación familiar a todas las familias, implementando así lo que Bate (2016) denomina 'servicios universales progresivos': servicios en los que existe un paquete básico - de cobertura universal-que se complementa con servicios de mayor intensidad cuando los/as profesionales valoran que una unidad familiar puede beneficiarse de ellos. El Programa de Apoyo Parental9 implantado en los países nórdicos (Marklund y Simic, 2012), los parenting shops ${ }^{10}$ que funcionan en las regiones

9 Un ejemplo de este tipo de intervención es el programa Apoyo Parental en la Región Nórdica (Parental Support in the Nordic Region), cuyo objetivo es que los padres y madres puedan acceder a servicios de apoyo familiar de forma fácil y desde una edad temprana. El programa cuenta con tres niveles de atención. El primero es de cobertura universal e incluye un número limitado de consultas individuales para cada familia. El segundo nivel de atención se ofrece en talleres grupales e incluye la creación de un grupo de apoyo mutuo para padres y madres. Se desarrolla en forma de sesiones semanales, de entre dos y dos horas y media, durante diez o doce semanas, e incluye la realización de lecturas, grupos de discusión, juegos de rol y ejercicios que deben llevarse a cabo de una reunión a otra. El tercer nivel de atención se dirige a familias con hijos/as que presentan graves problemas de conducta, e incluye sesiones individuales de una hora u hora y media de duración con una terapeuta familiar. El número de horas se establece en función de las necesidades de la familia. El programa incluye formación en habilidades parentales y la realización de ejercicios prácticos entre las sesiones.

${ }^{10}$ Estos centros ofrecen servicios dirigidos a cualquier persona involucrada en la crianza de un menor de entre o y 18 años, así como a familias que están esperando un hijo/a. El servicio se estructura en un continuo de atención que abarca tres niveles de servicios:

- Servicios universales dirigidos a todas las familias y que incluyen puntos de información, folletos, libros, materiales interactivos, una página web y sesiones formativas en torno a la crianza y la parentalidad positiva.

- Servicios universales específicamente dirigidos y adaptados para determinados grupos sociales o culturales, como son las familias en situación de pobreza o las familias de origen extranjero. En este caso, los centros trabajan conjuntamente con otras organizaciones de acción social o cultural que ya están en contacto con estos colectivos, con el objetivo de promover el acceso e incrementar la asistencia de estas unidades familiares a este 
de Flandes y Bruselas (Eurochild, 2012) o los Sure Start children centers en el Reino Unido (Bate, 2016) constituyen ejemplos de servicios integrados que ofrecen información, orientación y formación en el ámbito de la parentalidad positiva a todas las familias.

Cuadro 2. Programa Incredible Years ${ }^{12}$ (Reino Unido)

El programa Incredible Years tiene como objetivo mejorar las habilidades parentales para la crianza de los/as hijos/as, promoviendo una parentalidad positiva.

\section{Grupo al que se dirige la intervención}

El programa se dirige a familias con hijos/as de entre o y 12 años de edad, y cuenta con módulos específicos dirigidos a los siguientes grupos de edad:

- Bebés (0-1 año).

- Niños/as (1-3 años).

- Preescolares (3-6 años).

- Niños/as en edad escolar (6-12 años).

Además, los distintos módulos cuentan también con versiones que complementan la intervención grupal con visitas domiciliarias para las familias con mayores dificultades.

\section{Planteamiento que subyace a la intervención}

Este programa de parentalidad positiva se centra en fortalecer el vínculo entre los menores y sus progenitores, así como en mejorar las interacciones entre ambos. Uno de los objetivos principales es reducir los comportamientos punitivos por parte de los padres - que tienden a reforzar el mal comportamiento de los y las menores-y sustituirlos por respuestas que promuevan las habilidades sociales, emocionales y lingüísticas de los/as hijos/as. Además, en los programas orientados a los menores en edad preescolar o escolar el programa hace un fuerte hincapié en la estimulación de las habilidades cognitivas y lingüísticas de los/as menores, con el objetivo de mejorar el rendimiento académico de los/as niños/as.

\section{Forma de provisión del programa}

El programa se estructura en sesiones grupales semanales de entre dos horas y dos horas y media de duración exclusivamente dirigidas a padres y madres -a excepción del programa para niñas y niños menores de un año, donde los progenitores acuden junto con sus bebés-.

El número de sesiones varía en función de la versión del programa que se aplique, la cual depende, a su vez, de la gravedad de los problemas de crianza o conducta mostrados por niñas y niños, y de su edad (véase la Tabla 1).

Tabla 1. Número de sesiones grupales organizadas, según grupo de edad y versión del programa

\begin{tabular}{l|c|c|c|c}
\hline \multirow{2}{*}{ Versión del programa } & \multicolumn{4}{|c}{ Grupo de edad } \\
\cline { 2 - 5 } & $0-1$ año & $1-3$ años & 3-6 años & 6-12 años \\
\hline Básica & $8-9$ & 12 & $18-20$ & $18-20$ \\
\hline Avanzada* & \multicolumn{4}{|c}{$9-12$} \\
\hline
\end{tabular}

* El programa avanzado se realiza tras completar el programa básico y se centra en el desarrollo de habilidades interpersonales, el manejo de la rabia y la depresión, y la resolución de problemas. Se dirige a familias con menores con un problema de conducta diagnosticada.

Todas las sesiones deben estar dinamizadas por dos profesionales y cuentan con la participación de entre diez y catorce padres y madres.

\section{Coste del programa}

Rhiannon et al. (2016) estiman que el coste medio de poner en marcha el programa básico dirigido a niños/as de entre 3 y 6 años es de 2,418 $f$ por niño/a si los grupos son de ocho personas, y de 1,612 $f$ si los grupos se amplían hasta las doce personas.

\section{Resultados}

El programa Incredible Years ha sido ampliamente evaluado en distintos países. Un reciente metanálisis (Menting et al., 2013, cit. en Axford et al., 2015) que recogía evidencias sobre 50 de estos estudios (4.745 participantes) halló resultados positivos vinculados a la reducción del comportamiento disruptivo de los y las menores (tamaño del efecto $=0,27$, $p<0,001$ ) y una mayor prevalencia del comportamiento prosocial (tamaño del efecto $=0,23, p<0,001$ ) entre los menores cuyos progenitores participaron en el programa. 


\section{Promoción de la salud física y mental de los y las menores}

Las desigualdades en el ámbito de la salud también están estrechamente relacionadas con la situación social de los y las menores. Por ello, es necesario articular servicios y prestaciones que permitan compensar las desigualdades en el acceso a servicios y equipamientos relacionados con la salud, pero también a estilos de vida saludables, abordando aspectos como la alimentación, la higiene, y la promoción de la actividad física y prevención del sedentarismo, entre otros.

Los principales programas implementados en este ámbito incluyen: ayudas para la adquisición de productos de alimentación e higiene infantil; ayudas para la adquisición de bienes y servicios relacionados con la salud (gafas, audífonos) que no están cubiertos por los sistemas de salud pública; y programas de prevención, detección precoz y educación en el ámbito de la salud (Cowley et al., 2013; Riera et al., 2013). Además, recientemente se ha apuntado a la importancia de detectar los problemas de salud mental (ansiedad, depresión, adicciones) de los padres y madres, debido a la fuerte influencia que tienen en el bienestar y desarrollo temprano de los niños y niñas (McDonald, Moore y Goldfeld, 2012).

Algunas iniciativas de interés en este ámbito se han desarrollado en el Reino Unido, donde en las dos últimas décadas se han implantado y extendido progresivamente al conjunto de la población dos servicios de cobertura estatal que vale la pena destacar. Por un lado, la integración de los servicios de salud infantil en los centros de atención temprana a la infancia (Sure Start children's centers), dirigidos a las familias con hijos/as de entre o y 8 años de edad y que reúnen en una misma ubicación física todos los servicios dirigidos a la primera infancia (servicios de información y orientación a las familias, servicios de cuidado, actividades lúdicas y educativas, servicios sociales y servicios de salud).

También la implementación de programas de visitas domiciliarias efectuadas por profesionales cualificados en salud infantil puede considerarse una buena práctica en este ámbito (Cowley et al., 2013). Inicialmente, estas visitas domiciliarias únicamente se efectuaban a los hogares en situación de riesgo, pero en el Reino Unido, desde 2015, son obligatorias para todas las familias con hijos/as menores de 2 años. De hecho, la versión básica del programa incluye un total de cinco visitas domiciliarias: una antes del nacimiento del bebé, una justo después del nacimiento, una tercera al cabo de seis u ocho semanas, la cuarta cuando el niño o niña cumple su primer año, y una última dos años o dos años y medio después del nacimiento (Bate, 2016).

Las funciones de los equipos que realizan las visitas domiciliarias incluyen valorar de forma global el bienestar y las necesidades de los menores, sus progenitores y el conjunto de la unidad familiar. Esto supone que no sólo asumen un rol como agentes de salud, sino también una importante función comunitaria, ofreciendo información, derivando a las familias a otros servicios cuando lo consideran apropiado y coordinándose con otros profesionales para ofrecer una respuesta integral a las necesidades de las familias (National Institute for Care and Health Excellence, 2014). El programa consta, además, de tres niveles de provisión (universal, universal plus, universal partnership plus), de modo que, cuando se detectan problemas relacionados con la salud o el bienestar de los/as niños/as, se intensifica la frecuencia de las visitas domiciliarias.

Pese a la menor implantación de los servicios de promoción de la salud en nuestro país, un programa de referencia en el terreno de la atención a las familias en situación de vulnerabilidad social como es el Programa Caixa Prolnfancia también cubre este ámbito. En este caso, se ofrecen dos tipos de ayudas: una para adquirir productos de alimentación e higiene destinados a niños/as de entre o y 3 años, y otra para la adquisición y mantenimiento de gafas y audífonos de los menores de la unidad familiar. No obstante, se identifica la necesidad de profundizar esta línea de trabajo e incluir acciones dirigidas a la promoción de hábitos y estilos de vida saludables y la prevención del consumo de sustancias adictivas, entre otros (Riera et al., 2013).

\section{Refuerzo educativo y mejora del rendimiento escolar de los y las menores}

La probabilidad de tener un bajo rendimiento escolar es mayor entre los niños y niñas procedentes de hogares con un bajo nivel socioeconómico (Assiego y Ubrich, 2015; Field, 2010; Waldfogel y Washbrook, 2010). Una de las intervenciones más habituales de cara a promover el éxito escolar de los y las menores con bajo rendimiento académico son los programas de educación compensatoria, que ofrecen apoyo académico suplementario a estudiantes con bajas cualificaciones.

Uno de los de mayor alcance implementados en nuestro entorno ha sido el Programa de Acompañamiento Escolar (PAE), en funcionamiento entre 2005 y 2012. Este programa, aplicado en el ámbito de la educación secundaria, ofrecía un mínimo de cuatro horas de clases de refuerzo semanales en grupos de entre 5 y 10 personas a alumnado con bajas cualificaciones o dificultades de aprendizaje. Las actividades realizadas tenían como objetivo estimular los hábitos de lectura, adquirir técnicas de estudio y mejorar las habilidades sociales de los y las menores. La evaluación realizada por García e Hidalgo (2016) muestra una mejora en las habilidades de lectura de los y las menores y una reducción del $5 \%$ en la probabilidad de situarse por debajo del percentil 25 en la distribución de notas del colegio. Resulta de interés señalar que los mayores beneficios se obtienen con alumnado procedente de entornos rurales, 
en comparación el situado en entornos urbanos. Finalmente, la evaluación muestra también que el programa debe aplicarse durante un mínimo de tres años consecutivos en un centro determinado para que se observen resultados positivos y estadísticamente significativos en el rendimiento escolar.

Cuadro 3. Subprograma de refuerzo educativo del Programa Prolnfancia de La Caixa

El programa Prolnfancia de La Caixa tiene como objetivo ofrecer actividades de refuerzo educativo a menores en situación de desventaja social que presentan dificultades de escolarización.

\section{Grupo al que se dirige la intervención}

La participación de niños y niñas en las actividades de refuerzo educativo desarrolladas en el programa está supedita a

los siguientes criterios:

- Tener entre 3 y 16 años (según el tipo de actividad).

- Presentar problemas de rendimiento escolar, incluidos el absentismo, el abandono prematuro y la falta de adaptación a la escuela.

- Tener un rendimiento escolar aceptable, pero con predictores de riesgo (desestructura familiar o antecedentes de

fracaso escolar en otros miembros de la familia) que aconsejen el refuerzo como medida preventiva.

- Presentar dificultades logopédicas y psicomotrices que condicionen los procesos de aprendizaje y desarrollo.

\section{Planteamiento que subyace a la intervención}

Las actividades de refuerzo educativo que integran el programa tienen como objetivo desarrollar tres funciones, que en ocasiones pueden ser complementarias: prevención, reeducación y refuerzo permanente. El enfoque preventivo se centra en apoyar al alumnado con contextos familiares muy desestructurados, donde la concurrencia de numerosos indicadores de riesgo aconseja realizar las intervenciones de forma preventiva. Las actividades reeducativas, sin embargo, se dirigen a atender dificultades específicas a partir de un diagnóstico psicopedagógico afinado. El objetivo es llegar a compensar las dificultades del alumno o alumna (bloqueos en alguna materia, mecánica de determinados procedimientos de lectura, cálculo), momento en que cesa la intervención. Finalmente, las actividades de refuerzo permanente ofrecen apoyo continuado al alumnado que presenta dificultades manifiestas para seguir los aprendizajes o que está en riesgo evidente de fracaso escolar.

\section{Forma de provisión del programa}

El subprograma de refuerzo educativo se articula en torno a los siguientes tipos de actividades:

- Aula abierta / aula de estudio (biblioteca). Espacio para hacer deberes, consultar dudas, aprender a organizarse, consulta y préstamo de libros, estimulación del aprendizaje. Este recurso se orienta principalmente a disponer de un entorno facilitador para realizar las tareas escolares, de aprendizaje y el estudio.

- Grupos de estudio asistido. Apoyo a alumnado con niveles y dificultades similares. Las ratios de tres a cinco alumnos/as se consideran las más adecuadas. Esta actividad se centra en apoyar las tareas escolares y el estudio, la resolución de dudas y dificultades de comprensión, el seguimiento de la escolarización y el control de agenda.

- Refuerzo individual. Apoyo personalizado en función de la especificidad de las necesidades detectadas. Dado el coste del recurso, requiere una evaluación psicopedagógica afinada.

- Equipo escolar. Ayudas individuales para la adquisición de equipo escolar dirigidas a familias que no pueden adquirirlo por su situación económica.

- Atención logopédica. Atención centrada en el diagnóstico y tratamiento de las alteraciones del lenguaje y de la comunicación en los niños y niñas con edades comprendidas entre los 3 y los 12 años.

- Apoyo psicomotriz. Actividades basadas en la educación psicomotriz de los niños y niñas con edades comprendidas entre los 3 y los 12 años.

Dependiendo del tipo de actividad de la que se trate y del perfil de las personas usuarias, estas actividades pueden desarrollarse en diversas entidades e instituciones: escuelas, centros abiertos, centros juveniles, centros comunitarios, centros de acción social, centros de día, centros residenciales y centros, o gabinetes profesionales.

\section{Resultados}

El estudio de evaluación de impacto elaborado por Riera et al. (2014), en el que se comparan los resultados de dos cursos escolares (2012-2013 y 2013-2014) de las personas participantes en las actividades del subprograma de refuerzo escolar Prolnfancia de La Caixa, muestra los siguientes resultados:

- Entre el 65\% (2013-2014) y el 68,2\% (2012-2013) de las personas participantes mejoraron su rendimiento académico tras participar en el programa.

- Entre el $47,5 \%$ y el 52,9\% lograron aprobar el curso académico.

- El efecto del programa es mayor en la población que cursa educación primaria que en el alumnado de educación secundaria y, en ambos casos, el alumnado de los primeros ciclos obtienen mejores resultados que sus homólogos/as de cursos superiores. Concretamente, en el curso 2013-2014 el 80,6\% del alumnado de primer ciclo de educación primaria que participó en el programa mejoró su rendimiento académico, frente a sólo el 57,7\% del alumnado del segundo ciclo de educación secundaria obligatoria (ESO). 
- El programa también muestra resultados positivos por lo que respecta a la participación e integración de las familias en la dinámica escolar. A partir de la percepción de los y las profesionales que trabajan con las familias, en el curso 2013-2014 se considera que la evolución del 39,6\% de las familias en este ámbito fue muy satisfactoria, y en el 33,8\% de los casos se valoró que había sido satisfactoria.

- En cuanto al itinerario académico escogido por el alumnado participante en el programa tras finalizar la ESO, los resultados muestran que el $41,2 \%$ de los y las menores que participaron en el curso 2013-2014 continuaron estudios de bachillerato, el 32,4\% se decantaron por un ciclo formativo y el 8,8\% cursaron alguna formación no reglada.

Otra estrategia para tratar de compensar las desventajas en el ámbito escolar de los y las menores procedentes de entornos socialmente desfavorecidos ha sido la creación de las Zonas de Educación Prioritaria (ZEP). Ésta ha sido una estrategia implementada en varios países de la Unión Europea como estrategia para luchar contra el abandono escolar prematuro y el fracaso escolar en zonas de bajo nivel socioeconómico y elevadas tasas de abandono escolar prematuro. Las zonas de educación prioritaria suponen, al menos, la adopción de las siguientes medidas:

- Implementar estrategias pedagógicas innovadoras que se adapten a las características del alumnado.

- Garantizar la continuidad del alumnado en los distintos niveles educativos, mediante la creación de redes de centros escolares de educación prioritaria que garanticen la admisión preferente de dicho alumnado.

- Desarrollar estrategias cooperativas entre los distintos agentes de la comunidad educativa (alumnado, padres y madres, profesores) y con profesionales de otros servicios comunitarios (servicios sociales, servicios de animación sociocultural), de cara a mejorar el clima y la convivencia escolar.

- Reducir el número de alumnos por clase.

- Establecer la adquisición de habilidades comunicativas y de escritura avanzadas como la principal prioridad educativa de los centros.

- Crear un equipo de orientación y coordinación pedagógica que asesore y apoye a los profesores de los centros y promueva la coordinación pedagógica y la transmisión de buenas prácticas entre los centros pertenecientes a una misma red de educación prioritaria.

- Integrar el desarrollo de actividades socioculturales y deportivas en los centros fuera del horario escolar.

Las evaluaciones han demostrado que este tipo de intervenciones tienen una escasa eficacia cuando se aplican a gran escala (Inspection Générale de l'Education Nationale, 2006) y, de hecho, países como Francia y el Reino Unido, que habían aplicado este tipo de políticas en los años ochenta y noventa han pasado a centrarse nuevamente en la atención individualizada de los y las menores en riesgo de abandono escolar (Étienne, 2008). No obstante, existen evidencias de que la aplicación de este tipo de intervención sí puede resultar efectiva a pequeña escala y, especialmente, de cara a intervenir en centros escolares con una alta concentración de problemas educativos y sociales.

Un ejemplo de este tipo de intervención es el proyecto Bultzatzen, aplicado en dos centros de educación secundaria del municipio de Erandio (Bizkaia) desde 2004 y que ha apostado por la colaboración interinstitucional como forma de abordar, de manera integral, los problemas socioeducativos que afectan a buena parte del alumnado de dichos centros (Santibáñez y Maiztegui, 2013). La estrategia adoptada ha sido la integración de los miembros del Equipo de Intervención Socioeducativa con Infancia, Juventud y Familia - adscrito a los servicios sociales municipales - a la estructura de los centros, creando un comité coordinador encargado de diseñar, planificar y evaluar los proyectos y las iniciativas socioeducativas que se llevan a cabo en los centros. Por otro lado, también se ha optado por incluir a personal de este equipo entre los/as educadores/as encargados/as de ofrecer actividades extraescolares y clases de refuerzo al alumnado más allá del horario escolar. De este modo, se normaliza la presencia de los educadores en los centros. El programa Bultzatzen incluye actividades transversales curriculares para la prevención de la violencia entre iguales, la discriminación, el consumo de drogas y otro tipo de problemas escolares durante el horario lectivo, y ofrece un programa de clases de refuerzo diarias para el alumnado con bajo rendimiento académico, que se combina con actividades de ocio y tiempo libre. El servicio educativo complementario abarca la franja horaria entre las 16' 45 y las 19'30, y se ofrece diariamente. Por otro lado, el proyecto también trata de mejorar el rendimiento y la integración escolar de los y las menores a través de la implicación de los padres y madres en la vida del centro, incluyendo actividades participativas para recoger sus demandas, necesidades y aspiraciones con relación a la educación de sus hijos/as y responder a ellas de la mejor manera posible (Santibáñez y Maiztegui, 2013).

Un tercer tipo de intervenciones que han demostrado su eficacia para mejorar el rendimiento educativo de los menores en riesgo se centran en motivar y capacitar a los progenitores para apoyar y acompañar los procesos de aprendizaje y éxito escolar de sus hijos/as (Goodall, 2013; Allen, 2011; Waldfogel y Washbrook, 2011). A partir de la revisión de intervenciones dirigidas a familias con niños/as de entre 5 y 19 años de edad, Goodall identifica seis factores de éxito en este tipo de programas: 
- Promover el acceso a servicios de educación infantil de calidad, así como la participación en actividades de aprendizaje en familia desde una edad temprana (grupos de lectura, espacios de juego familiar, organización de actividades lúdicoeducativas dirigidas a familias).

- Mejorar el entorno de aprendizaje en el hogar: promover la creación de entornos ordenados y estructurados, el mantenimiento de rutinas en la organización de la vida familiar, y el acceso a materiales y equipamientos educativos (libros, ordenador, juguetes didácticos) que faciliten la creación de rutinas de estudio en el hogar. Este tipo de programas suelen estructurarse a partir de visitas domiciliarias y sesiones grupales donde se capacita a padres y madres para crear un entorno de estudio adecuado en el hogar y apoyar a sus hijos/as en la realización de los deberes escolares (Brooks et al., 2008).

- Mantener la participación y el interés de padres y madres en la educación de sus hijos/as a lo largo de toda la trayectoria escolar. Se observa que en los niveles de educación infantil y primaria se hace un énfasis mucho mayor en la contribución de los padres y madres al éxito escolar de sus hijos/as, en comparación con la educación secundaria, una etapa especialmente crítica por lo que respecta al abandono escolar prematuro. En este sentido, se recomienda continuar organizando actividades y programas que promuevan esta participación y muestren a padres y madres de qué forma pueden apoyar la trayectoria escolar de sus hijos/as en esta etapa educativa (Waldfogel y Washbrook, 2011).

- Promover que los padres y las madres tengan y sostengan elevadas aspiraciones con relación al rendimiento académico de sus hijos/as. Diversos estudios muestran que los padres y madres de bajo nivel socioeconómico tienden a mantener aspiraciones más bajas con relación al rendimiento escolar de sus hijos/as (Goodall, 2013).

- Promover que los padres y madres participen activamente en la educación de sus hijos/as. Diversas investigaciones muestran que el interés y la implicación directa de padres y madres en la realización de los deberes y otras actividades educativas de los hijos/as mejora de forma sustancial las posibilidades de éxito escolar (Goodall, 2013).

\section{La participación en actividades culturales, de ocio y tiempo libre}

Las actividades culturales, de ocio y tiempo libre juegan un papel decisivo en la formación de experiencias tempranas en la infancia, la ampliación de las oportunidades de aprendizaje y la adquisición de nuevas experiencias, al tiempo que permiten expandir las redes sociales de niños y niñas más allá de sus contextos habituales (Scott, 2013).
En un informe sobre el derecho al ocio educativo de niños y niñas elaborado por el Síndic de Greuges de Catalunya (2014), este organismo alertaba sobre la falta de regulación expresa de este derecho en la mayoría de contextos normativos y el acceso desigual que ello conlleva para los y las menores en situación de desventaja social. Entre las recomendaciones realizadas por este organismo, se incluyen las siguientes:

- Desarrollar una norma que regule el derecho de los niños y niñas al ocio educativo en condiciones de igualdad.

- Crear un sistema de ayudas económicas (ayudas económicas directas, bonificaciones y exenciones, sistemas de tarifación social) que promueva el acceso de los y las menores en situación de desventaja a las actividades extraescolares, las actividades complementarias (excursiones y colonias, tanto dentro como fuera del periodo escolar) y otras actividades socioeducativas que se desarrollen en su comunidad fuera del ámbito escolar.

- Diseñar e implementar planes locales de dinamización del ocio educativo, especialmente en municipios o zonas del territorio socialmente desfavorecidas, a fin de combatir las desigualdades territoriales en la provisión y acceso a este tipo de recursos. Algunas acciones fundamentales de estos planes deben ser: establecer medidas de fomento de la participación de colectivos específicos en las actividades de deportivas, de ocio y tiempo libre ofertadas en el municipio; incluir programas y actuaciones de apoyo dirigidos a los y las adolescentes en situación de riesgo social; favorecer el asociacionismo juvenil basado en el voluntariado y en proyectos de autogestión de grupos de jóvenes; y, finalmente, promover la coordinación y el trabajo integrado entre ayuntamientos, centros escolares y entidades que desarrollan actividades de ocio educativo para apoyar la participación de los niños y niñas en situación de vulnerabilidad en este tipo de actividades.

- Reconocer y promover a los agentes de ocio educativo que operan en el territorio.

Una buena práctica en este ámbito son las escuelas de verano que aúnan clases de refuerzo escolar y las actividades lúdicas y deportivas para alumnado con bajo rendimiento académico en situación de desventaja social. Si bien estos programas resultan adecuados para combatir el fracaso escolar y promover, al mismo tiempo, el acceso a actividades de ocio y tiempo libre, también pueden dificultar la participación en otros espacios de ocio educativo, dando lugar a cierta segregación (Giannaka et al., 2007). Por eso, se recomienda diseñar estas actuaciones con un carácter transversal, centrado también en garantizar el acceso a los servicios deportivos, culturales y de ocio y tiempo libre ofertados en la comunidad. 


\section{Los servicios integrados de apoyo a las familias}

Además de sobre el contenido de los programas y servicios mencionados, existe también un debate sobre la forma de provisión y el tipo de cobertura que éstos deben ofrecer para garantizar un buen acceso a la población en vulnerabilidad social. A este respecto, hay tres cuestiones que deben tenerse en cuenta. En primer lugar, la integración de los servicios dirigidos a atender a la infancia y a sus familias. En segundo lugar, el acceso universal o específico a los servicios. Y en tercer lugar, si los servicios se dirigen exclusivamente a atender las necesidades de los y las menores o si, por el contrario, también incluyen programas que tienen como objetivo responder, en mayor o menor medida, a las necesidades de sus progenitores.

Con relación al carácter integrado o fragmentado de los servicios, existe una evidencia científica creciente de que los servicios integrados aumentan el acceso de las poblaciones más vulnerables a los recursos disponibles, contrarrestando parcialmente la escasa demanda de servicios de estos grupos de población (OCDE, 2015). A este respecto, cabe destacar que en las últimas décadas se ha extendido en Europa la creación de centros integrales de apoyo a la infancia y a las familias, que están ofreciendo buenos resultados de cara a mejorar el acceso a los servicios de los grupos de población vulnerable (Frazer, 2016).

Este tipo de centros presta un amplio abanico de servicios (educativos, de salud, de cuidado, programas de parentalidad positiva, programas de inserción sociolaboral) dirigidos a las familias. Los distintos modelos varían en cuestiones clave, como el rango de edad de los y las menores a los que se dirigen, el tipo de servicios ofertados, la intensidad de la atención o los requisitos de acceso. En cualquier caso, la presencia de estos centros se ha incrementado de forma progresiva, y actualmente este modelo de servicio está presente en países tan dispares como Suecia, Dinamarca, Bélgica, Canadá, República Checa, Francia, Alemania, Italia, los Países Bajos, Polonia, Eslovenia y el Reino Unido, si bien es cierto que no en todos los casos son servicios universales, ni están implantados a escala estatal (Frazer, 2016; OCDE, 2015).
En cuanto a la universalidad de los servicios, la mayoría de las evaluaciones realizadas apuntan a que los servicios universales únicamente logran responder de forma satisfactoria a los problemas leves de las familias vulnerables, mientras que ofrecen peores resultados que los servicios específicos cuando se trata de abordar necesidades o problemas complejos. En este sentido, la literatura especializada hace dos recomendaciones. Por un lado, destaca la importancia de mantener el modelo de gestión de caso habitualmente utilizado en los servicios sociales para atender a estas familias -independientemente de si los servicios son de carácter universal o específico-, de modo que se garantiza la presencia de un profesional de referencia encargado de coordinar la atención y las comunicaciones con dicha unidad familiar. Por otro lado, señala el modelo en cascada como una modalidad de prestación de servicios que resulta eficaz a la hora de aunar los servicios universales con la atención específica a los grupos de población más vulnerable.

Este modelo ha sido aplicado, sobre todo, en Suecia y Dinamarca - aunque recientemente otros países han adoptado también fórmulas similares-, y la idea subyacente es ofrecer una serie de servicios básicos de carácter universal combinados con una oferta de servicios especializados y adaptados a las necesidades de las familias más vulnerables. Cuando se detecta la necesidad de recibir atención especializada en una unidad familiar, se la deriva a estos recursos, garantizando así la calidad y la continuidad de la atención en un mismo circuito asistencial (OCDE, 2015).

Finalmente, también es importante considerar si los servicios de refuerzo a la infancia deben centrarse sólo en mejorar el bienestar de los y las menores, o si, por el contrario, deben considerar las necesidades de la unidad familiar desde un punto de vista global. La evidencia científica a este respecto señala la conveniencia de incorporar algunas de las necesidades o preocupaciones de los progenitores en el diseño los servicios y la oferta asistencial. En este sentido, existen evidencias que muestran que el hecho de que los progenitores reciban servicios específicos para atender algunas de sus demandas mejora la relación de las familias con los servicios, al tiempo que permite incrementar el acceso a aquéllos para los grupos de población más vulnerables (OCDE, 2015; Goodall, 2013; Goodall y Vorhaus, 2012). 


\section{Bibliografía referenciada}

ALLEN, G. (2011): Early Intervention: The Next Steps. An Independent Report to Her Majesty's Government, Londres, HM Government [<http:// www.siis.net/es/documentacion/catalogo/ Record/184216>].

ASSIEGO, V.; y UBRICH, T. (2015): Iluminando el futuro. Invertir en educación es luchar contra la pobreza infantil, Save The Children [shttp:// www.siis.net/es/documentacion/catalogo/ Record/499086>].

AXFORD, N. et al. (2015): The Best Start at Home. What Works to Improve the Quality of Parentchild Interactions from Conception to Age 5 Years? A Rapid Review of Interventions, Early Intervention Foundation [<http://www.eif.org. uk/wp-content/uploads/2015/03/The-BestStart-at-Home-report1.pdf〉].

BATE, A. (2016): Early Intervention, serie Briefing Papers, no 07647 , House of Commons Library [<http:// researchbriefings.files.parliament.uk/ documents/CBP-7647/CBP-7647.pdf)].

BROOKS, G. et al. (2008): Effective and Inclusive Practices in Family Literacy, Language and Numeracy: A Review of Programmes and Practice in the UK and Internationally, serie Reseach Papers, CfBT Education Trust [<https://www. educationdevelopmenttrust.com/ /media/ cfbtcorporate/files/research/2008/r-effectiveand-inclusive-practices-in-family-literacyreview-2008.pdf $>$ ].

COWLEY, S. et al. (2013): Why Health Visiting? A Review of the Literature about Key Health Visitor Interventions, Processes and Outcomes for Children and Families, Londres, King's College London [rhttps://www.kcl.ac.uk/nursing/ research/nnru/publications/Reports/WhyHealth-Visiting-NNRU-report-12-02-2013.pdf `].
ÉTIENNE, R. (2008): Educational Policies that Address Social Inequality. France Case Study Report 2. Priority Education Zones (ZEP) and Priority Education Networks (REP), EPASI [<http://archive. londonmet.ac.uk/epasi.eu/CaseStudyFR2. pdf〉].

EUROCHILD (2012): Early Intervention and Prevention in Family and Parenting Support. Compendium of Inspiring Practices, Bruselas, Eurochild [<http://www.siis.net/es/documentacion/ catalogo/Record/189441>].

FIELD, F. (2010): The Foundation Years: Preventing Poor Children Becoming Poor Adults. The Report of the Independent Review on Poverty and Life Chances, Londres, HM Government [<http:// www.siis.net/es/documentacion/catalogo/ Record/179786〉].

FRAZER, H. (2016): Prevention and Early Intervention Services to Address Children at Risk of Poverty. Peer Review in Social Protection and Social Inclusion. Synthesis Report, Bruselas, Comisión Europea [shttp://www.siis.net/es/ documentacion/catalogo/Record/517159'].

FUNDACIÓN FOESSA (2016): La transmisión intergeneracional de la pobreza: factores, procesos y propuestas para la intervención, Madrid, Fundación Foessa [<http://www. siis.net/es/documentacion/catalogo/ Record/510327〉].

GARCÍA-PÉREZ, J.; y HIDALGO-HIDALGO, M. (2016): No Student Left Behind? Evidence from the Programme for School Guidance in Spain, serie Fedea Policy Papers, no 2016/20 [rhttp:// documentos.fedea.net/pubs/fpp/2016/09/ FPP2016-20.pdf〉]. 
GIANNAKA, G. et al. (2007): Educational Policies that Address Social Inequality. Cyprus Case Study Report 1. Education Priority Zones (ZEP), EPASI [<http://archive.londonmet.ac.uk/epasi.eu/ CaseStudyCY1.pdf〉].

GOFF, J. et al. (2013): Evaluation of Children's Centres in England (ECCE). Strand 3: Delivery of Family Services by Children's Centres. Research Report, University of Oxford [<https://www. gov.uk/government/uploads/system/uploads/ attachment_data/file/224096/DFE-RR297.pdf>].

GOODALL, J. (2013): “Parental engagement to support children's learning: A six point model”, School Leadership \& Management, no3, págs. 133150 [rhttps://doi.org/10.1080/13632434.2012 $.724668>]$.

GOODALL, J.; y VORHAUS, J. (2012): Review of Best Practice in Parental Engagement, serie Research Report, no DFE-RR156, Department for Education [<https://www.gov.uk/government/ uploads/system/uploads/attachment_data/ file/182508/DFE-RR156.pdf〉].

HARVEY, B. (2014): The Case for Prevention and Early Intervention. Promoting Positive Outcomes for Children, Families and Communities, Dublín, Prevention and Early Intervention Network [rhttps://www.tcd.ie/Education/assets/ documents/PEIN\%20A4\%20Report-April\%20 2014.pdf>].

INSPECTION GÉNÉRALE DE L'ÉDUCATION NATIONALE (2006): La contribution de l'éducation prioritaire à l'égalité des chances des élèves, serie Rapports, $n^{\circ}$ 2006-076, Ministère de l'Éducation Nationale, de l'Enseignement Supérieur et de la Recherche [<http://media. education.gouv.fr/file/35/7/3357.pdf >].

KAROLY, L.; KILBURN, M. R.; y CANNON, J. (2005): Early Childhood Interventions: Proven Results, Future Promise, serie Monographs, Santa Monica, Rand Corporation [<http://www.rand.org/pubs/ monographs/MG341.html>].

MARKLUND, K.; y SIMIC, N. (2012): Nordic Children. Early Intervention for Children and Families, Nordic Centre for Welfare and Social Issues [rhttp:// www.siis.net/es/documentacion/catalogo/ Record/200400〉].

MCDONALD, M.; MOORE, T. G.; y GOLDFELD, S. (2012): Sustained Home Visiting for Vulnerable Families and Children: A Literature Review of Effective Programs, Parkville, The Royal Children's Hospital Centre for Community Child Health; Murdoch Childrens Research Institute [shttp://www.rch.org.au/uploadedFiles/Main/ Content/ccch/resources_and_publications/ Home_visiting_lit_review_programs_revised Nov2012(1).pdf)].

NATIONAL INSTITUTE FOR CARE AND HEALTH EXCELLENCE. (2014): Health Visiting, serie NICE Local Government Briefings National Institute for Health and Care Excellence [rhttps://www.nice. org.uk/guidance/lgb22/resources/healthvisiting-60521203534021)].

OCDE (2015): "Integrating service delivery for vulnerable families. Preventing disadvantage?”, en OCDE, Integrating Social Services for Vulnerable Groups: Bridging Sectors for Better Service
Delivery, París, OECD Publishing [<https:// dx.doi.org/10.1787/9789264233775-5-en>].

RIERA, J. et al. (2013): Programa Caixa Proinfancia. Modelo de promoción y desarrollo integral de la infancia en situación de pobreza y vulnerabilidad social, Barcelona, Obra Social La Caixa [<http://www.siis.net/es/ documentacion/catalogo/Record/181630>].

RODRIGO LÓPEZ, M. J.; MÁIQUEZ CHAVES, M. L.; y MARTÍN QUINTANA, J. C. (2010): La educación parental como recurso psicoeducativo para promover la parentalidad positiva, Madrid, Federación Española de Municipios y Provincias [<http:// www.msssi.gob.es/ssi/familias/nfancia/docs/ eduParentalRecEducativo.pdf〉].

SAMMONS, P. et al. (2015): The Impact of Children's Centres: Studying the Effects of Children's Centres in Promoting Better Outcomes for Young Children and Their Families. Evaluation of Children's Centres in England (ECCE, Strand 4). Research Report, Oxford, University of Oxford [rhttps:// www.gov.uk/government/uploads/system/ uploads/attachment_data/file/485346/DFERR495_Evaluation_of_children_s_centres_in_ England_the_impact_of_children_s_centres. pdf〉].

SANTIBÁÑEZ, R.; y MAIZTEGUI, C. (2013): “Bultzatzen - Animando al Éxito, una experiencia socioeducativa y de cooperación interinstitucional en la escuela", Revista de Educación Social, no 16 [rhttp://www.siis.net/ es/documentacion/catalogo/Record/191956)]

SCOTT, D. (2013): “Economic inequality, poverty, and park and recreation delivery", Journal of Park and Recreation Administration, vol. 31, nº 4, págs. 1-11 [rhttp://people.tamu.edu/ dscott/340/ U3\%20Readings/3\%20-\%202013\%20JPRA\%20 -\%20Economic\%2olnequality.pdfı].

SÍNDIC DE GREUGES DE CATALUNYA (2014): Informe sobre el derecho de los niños al ocio educativo y a las salidas y colonias escolares, Síndic de Greuges de Catalunya [<http://www.sindic.cat/site/ unitFiles/3687/Informe\%20sobre\%20el\%20 ocio\%2oeducativo.pdf $>$.

STORMSHAK, E. A.; CONNELL, A.; y DISHION, T. J. (2009): "An adaptive approach to family-centered intervention in schools: Linking intervention engagement to academic outcomes in middle and high school", Prevention Science: The Official Journal of the Society for Prevention Research, vol. 10, págs. 221-235 ['https://doi. org/10.1007/s11121-009-0131-3/].

UNICEF (2014): La infancia en España. El valor social de los niños: hacia un pacto de Estado por la infancia, Madrid, Unicef Comité Español [<http:// www.siis.net/es/documentacion/catalogo/ Record/198602>].

WALDFOGEL, J.; y WASHBROOK, E. (2011): “Early years policy”, Child Development Research, vol. 2011, 343016 [/https://doi. org/10.1155/2011/343016)].

- (2010): Low Income and Early Cognitive Development in the U.K. A Report for the Sutton Trust [<http://www.suttontrust.com/wp-content/ uploads/2010/02/Sutton_Trust_Cognitive_ Report.pdf $\rangle$. 